\title{
Evaluation of illustration-inspired techniques for time-varying data visualization
}

\author{
Alark Joshi ${ }^{\dagger 1}$ and Penny Rheingans ${ }^{\ddagger 1}$ \\ ${ }^{1}$ Computer Science Department, University of Maryland Baltimore County
}

\begin{abstract}
Illustration-inspired techniques have provided alternative ways to visualize time-varying data. Techniques such as speedlines, flow ribbons, strobe silhouettes and opacity-based techniques provide temporal context to the current timestep being visualized. We evaluated the effectiveness of these illustrative techniques by conducting a user study. We compared the ability of subjects to visually track features using snapshots, snapshots augmented by illustration techniques, animations, and animations augmented by illustration techniques. User accuracy, time required to perform a task, and user confidence were used as measures to evaluate the techniques. The results indicate that the use of illustration-inspired techniques provides a significant improvement in user accuracy and the time required to complete the task. Subjects performed significantly better on each metric when using augmented animations as compared to augmented snapshots.
\end{abstract}

Categories and Subject Descriptors (according to ACM CCS): H.1.2 [Human factors]:

\section{Introduction}

Illustration-inspired techniques have been found to be effective at conveying information succinctly and effectively. Techniques inspired by photography as well as illustrations have been adapted to visualizing time-varying data [WS03, SJEG05]. Practitioners currently use large panoramas of snapshots (as shown in Figure 1) taken over time or watch an animation of these snapshots to perform feature tracking. However, as can be seen by looking at the series of snapshots as shown in Figure 1, it is very hard to identify the direction in which the contained three-dimensional features are moving.

To measure the visual tracking ability of human observers, Pylyshyn [Pyl03] showed subjects a series of moving objects over time. Subjects were asked to visually track these objects as they moved. The study found that a human observer can successfully track up to five objects moving at a relatively moderate speed, but as the number of moving objects increased, the user's ability to track the features decreased

\footnotetext{
† Alark Joshi: alark1@cs.umbc.edu

‡ Penny Rheingans: rheingan@cs.umbc.edu
}

dramatically. He also found that the user's ability to track features dropped sharply as the speed of the moving objects increased.

In previous work, we proposed the use of techniques inspired by comics to augment time-varying data visualizations with illustrative cues to provide temporal context [JR05]. We presented four techniques: speedlines, flow ribbons, strobe silhouettes and opacity modulation techniques to convey positional change in time-varying data. It was claimed that the use of illustration-inspired techniques would aid the visual feature tracking abilities in visualizing time-varying data.

We evaluated the effectiveness of the illustration-inspired techniques by conducting a formal user study. The techniques were evaluated using three measures: accuracy in completing a task, time required by the users to complete a task and user confidence. Illustration-inspired techniques can be classified into two groups: Speedlines and flow ribbons are similar in nature and opacity modulation and strobe silhouettes are similar in the way they communicate path positions of features. We evaluated speedlines and opacitybased techniques, since they were representative of these two classes of illustration-inspired techniques. 

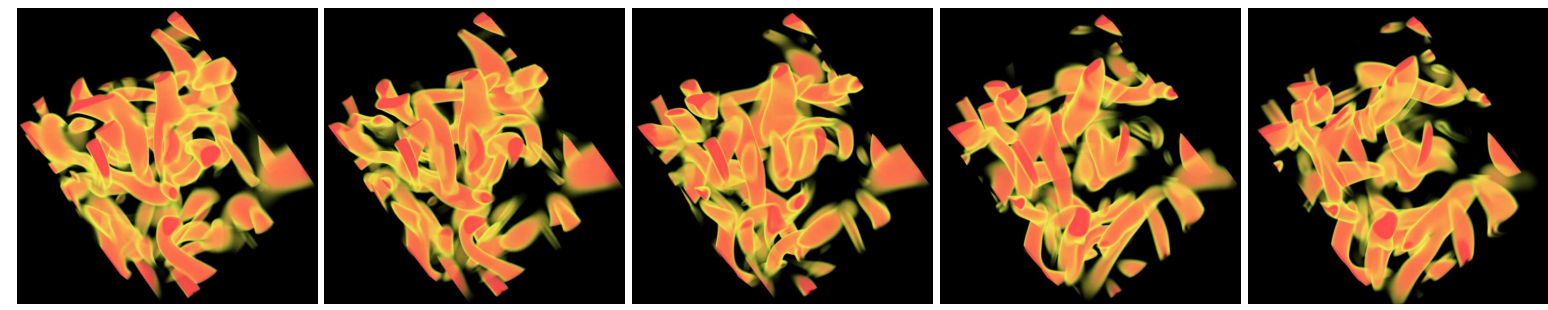

Figure 1: These are a set of snapshots from evenly spaced time steps of the turbulent vortex dataset [FS98]. As is evident from looking at the snapshots, it is very hard to correlate and track a particular feature over different time steps. These images were generated using Volview [Kit06].

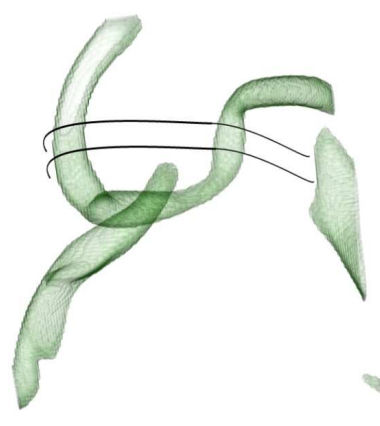

Figure 2: The image depicts change over time in a feature for CFD data. The rightward motion of the flow feature is conveyed using speedlines.

\subsection{Speedlines}

Speedlines are defined as lines that convey information to the viewer about the path traversed by a particular feature over time. They are basically lines that follow a particular feature over time. Illustrators have used speedlines to convey motion by altering the characteristics of these lines. The thickness, line style, and variation of the line's opacity are among the characteristics that successfully convey change in direction. Darker, thicker regions of the line convey an older time step whereas lighter, thinner regions of the speedline depict a more recent time step.

In our study, the speedlines technique was applied to synthetic data as well as real-world data from the computational fluid dynamics (CFD) domain. In Figure 2, the speedlines depict the rightward motion of the flow feature over the underlying features.

\subsection{Opacity modulation}

Illustrators often used blurred, desaturated images to depict older time steps with brighter, more detailed images representing newer time steps. In visualization, the same effect can be obtained by using opacity modulation techniques.

For this technique, an illustration feature is identified and

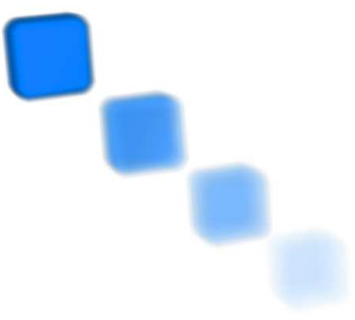

Figure 3: The image conveys change over time using opacity-based techniques. The upward motion of the feature is conveyed using opacity-based techniques. The older timesteps, shown by the faded representations of the feature over time, provide temporal context.

opacity-modulated snapshots of each timestep are merged into one visualization. Older timesteps are represented by translucent, blurred representations while newer timesteps are more crisp and brightly displayed. This provides insight into the origin of the feature and its path through multiple timesteps.

This technique was applied to synthetic data as well as real-world data. Figure 3 shows a visualization where the older time step is shown by a translucent, faded representation, whereas the newer time step is crisp and brighter. This visualization conveys the upward leftward motion of the feature.

\section{Hypothesis}

Our testable hypothesis is that illustration-inspired techniques can lead to improved visual tracking of features as they move over time. By improved visual tracking, we mean that the techniques will facilitate faster and more accurate visual tracking than standard snapshot-based or animationbased visualization of time-varying data. 


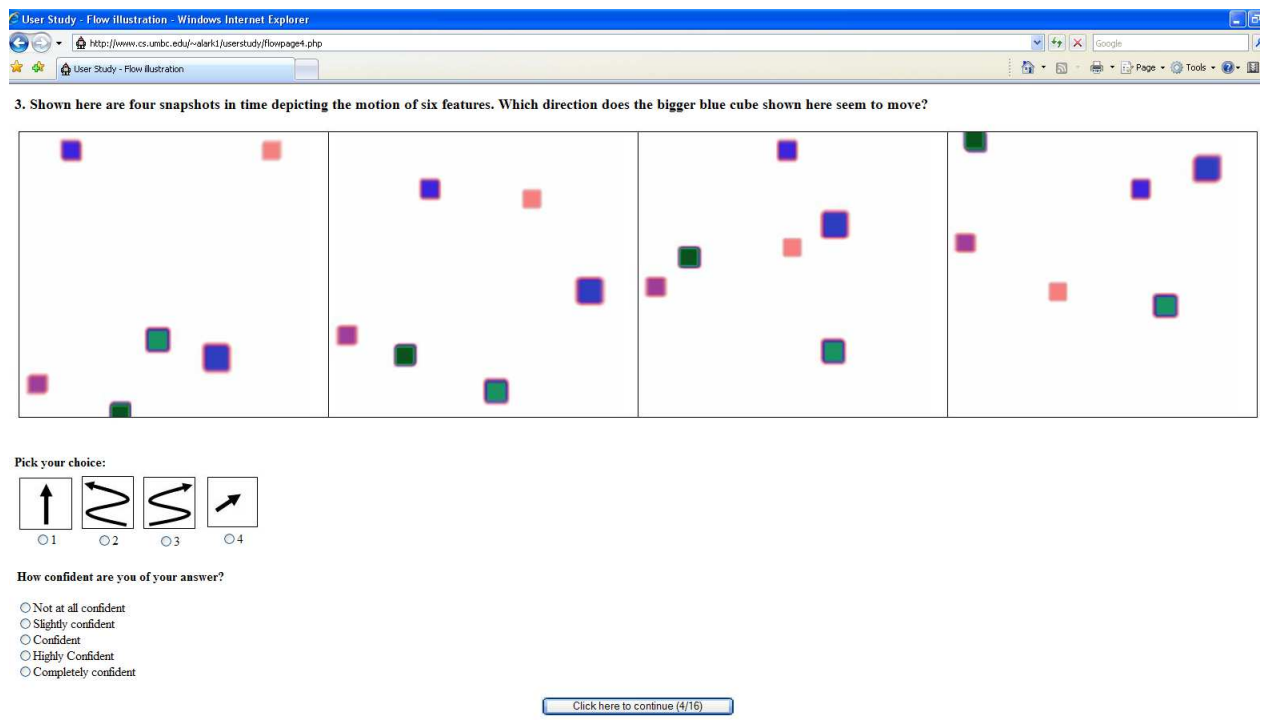

Figure 4: A screenshot of a sample screen that is shown to the subjects. The subjects were shown snapshots and were asked to indicate the direction of motion of a particular feature moving over time. They were also requested to specify a confidence level in their answer.

\section{Independent variable}

The independent variable for the user study is the visualization technique being used to visualize the time-varying data:

- A panorama of snapshots (such as the one shown in Figure 1).

- A single snapshot of the current timestep augmented with one of the illustration-inspired techniques. Figure 2 shows an example image of the same.

- An animation depicting the motion of the feature over time.

- An animation augmented with one of the illustrationinspired techniques.

\section{User study details}

Before we began the formal evaluation process, we ran a pilot experiment. The pilot study was not timed or scored for user accuracy. The pilot study was conducted with three subjects whose answers were not considered in the final evaluation of the techniques.

\subsection{Subjects}

We tested the illustration-inspired techniques with 24 subjects (15 males and 9 females aged 22 to 30) who had basic familiarity with using computers. We performed full factorial, within-subjects testing to evaluate the techniques. In order to balance ordering effects, we tested the subjects with all possible combinations of orderings of trials.

\subsection{Datasets}

Both synthetic and real-world data were presented in all four representations. The synthetic datasets that we used consisted of either a single feature tracing a simple path (linear or circular), multiple features tracing simple paths (linear, circular, spiral) and multiple features tracing complex paths.

The real world data that we used is the turbulent vortex dataset from Rutgers University [FS98]. The dataset is a pseudospectral simulation of coherent turbulent vortex structures with a $128 \times 128 \times 128$ resolution (100 time steps). The variable being visualized is vorticity magnitude. The dataset has numerous features that change position over time.

\subsection{Procedure}

During the course of conducting the user study, we first explained the study procedure to the subject. On obtaining consent from the subject for the study, we conducted the user study. At the end of the user study, we requested the subjects to fill out a usability questionnaire to get subjective feedback from them.

The user study was conducted using a web browser. Since we had to show videos to the subjects, we used Riva Free FLV encoder to encode AVI files into flash files that can be shown to the viewer in the browser setting.

Figure 4 shows a screenshot of the snapshot-based visualization that we showed the subjects. Figure 5 shows a screenshot of the augmented snapshots technique that was shown to the subjects. We first asked the user a question and depending on the kind of data (snapshots or animation), the 
7. Shown here is one snapshot in time depicting the motion of three features. Which direction does the middle object shown here seem to move?

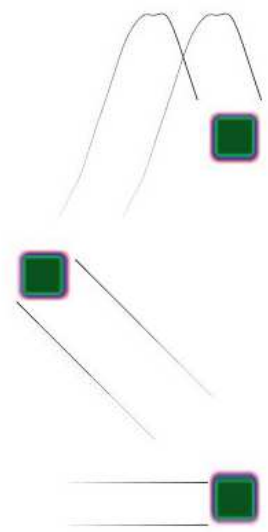

Pick your choice:

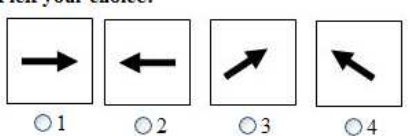

How confident are you of your answer?

Not at all confident

Slightly confident

Confident

OHighly Confident

Completely confident

Figure 5: This is a screenshot showing an augmented snapshot based visualization to the subjects. The users were asked to indicate the direction of motion as well as their confidence in their answers.

user observed it and provided an answer. We asked the user to specify the direction in which a particular feature was moving. The user picked one of the given choices and indicated a confidence level in their answer. We used a Likert scale to measure the confidence that the subjects had in their answers. The user was asked to select a confidence level from one of: Not at all confident, Slightly confident, Confident, Highly Confident, and Completely confident.

\subsection{Questionnaire}

Subjects were requested to fill out a questionnaire evaluating the effectiveness of the various techniques. Their answers were obtained on a Likert scale of 1 (easy/agree) to 9 (hard/disagree). The questions asked in the questionnaire were as follows:

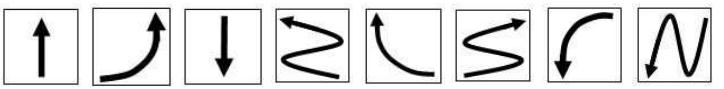

Figure 6: These images show a subset of the kinds of motion that we asked the subjects to choose. This enabled us to test more complex motions than just simple leftwards, rightwards kinds of motion.

1. Were the questions asked for each evaluation straightforward?

2. Overall, did you think the speedlines techniques helped convey direction better than standard visualization techniques?

3. Overall, did you think the opacity-based techniques helped convey direction better than standard visualization techniques?

4. Could you perform simple tasks such as tracking a single feature using standard snapshots or animation-based techniques?

5. Could you perform simple tasks such as tracking a single feature using illustration-based techniques?

6. Could you perform hard tasks such as tracking multiple features using standard snapshots or animation-based techniques?

7. Could you perform hard tasks such as tracking multiple features using illustration-based techniques?

\section{Tasks}

We asked users to track features as they were moving over time. Such tasks are representative of what researchers need to do on a regular basis as they track vortex tubes in feature data, as they track hurricane features over time, as they track the energy of jets entering a region, and so on. Our tasks were simple enough to test the effectiveness of the illustration-inspired techniques with subjects who are not necessarily application-domain experts; nevertheless, they represent tasks that scientists need to perform on a regular basis. The task-based question that we asked the user was Which of these paths seems to best represent the observed direction of motion of the feature?

The subject was presented with four choices of paths and was asked to select one of them according to the perceived motion. This enabled us to test complex motion paths instead of just simple linear motion of features. Figure 6 shows some of the images that we showed our subjects. They would indicate the perceived direction of motion of the feature by selecting one such glyph.

\section{Dependent variables}

We measured the accuracy of the subjects in performing the task in addition to the time required to complete the task. The user performance time is the time required by the user 
to read the question, look at the snapshots or animation and specify the answer. Additionally, we measured the confidence of the user in their answer. We also obtained feedback in the form of subjective satisfaction where we asked subjects to rate their experience on a scale of 1-9 (Likert scale).

\section{Results}

We analyzed the results of the user study using statistical techniques. To compare the four different visualization types, we used the statistical test Analysis of Variance (ANOVA) as well as Tukey's post-hoc pairwise comparison test . These tests allowed us to compare the accuracy, timings, and confidence obtained from the four groups (snapshots, augmented snapshots, animations, and augmented animations). The test began with a null hypothesis that the use of illustration-inspired techniques provided no speedup in completing tasks, no improvement in accuracy, and that the users felt equally confident in their answers for all techniques. The statistical measure of significance $p$ evaluates the probability of the result agreeing with the null hypothesis. For values of $p<0.05$, the null hypothesis is rejected, implying that the use of illustration-inspired techniques makes a difference.

The first metric that we used to evaluate the techniques was user accuracy, defined as the number of correct answers per user per technique. The mean and standard deviation for each technique are listed in Table 1. Figure 7 shows a graph of the same data. A comparison of the snapshots technique with the snapshots technique augmented with the illustration-inspired techniques shows that the subjects got more answers correct using the augmented snapshots. Similarly, in the case of animations compared to augmented animations, the subjects were more accurate when using augmented animations, as can be seen in Figure 7. The temporal context that the illustration-inspired techniques provide seem to help users complete the task more accurately. Amongst all the four techniques, the augmented animations techniques seems to provide users with the most useful information to correctly complete the task.

Analyzing the accuracy results using the ANOVA test yields the results shown in Table 2. The variation between the four different visualization techniques is high. The probability $p$ of this result assuming the null hypothesis is less than 0.0001. This implies that the result is extremely significant and that the null hypothesis is rejected. This proves that the use of illustration-inspired techniques increases the user accuracy for visual tracking of features.

To identify pairwise significance, the minimum pairwise difference was calculated using the Tukey test. The computed minimum pairwise difference was $D_{\min }=4.706$ with $Q_{t}=3$.9. For all values of difference of means larger than $D_{\min }$ the pairwise comparisons are significant $(p<0.05)$. As can be seen from Table 3, the results are significant when

\begin{tabular}{|c|c|c|}
\hline Type of visualization & Mean & Std. Dev. \\
\hline Snapshots & 76.851 & 10.288 \\
\hline Augmented Snapshots & 91.67 & 11.7284 \\
\hline Animations & 91.67 & 11.111 \\
\hline Augmented Animations & 97.22 & 5.144 \\
\hline
\end{tabular}

Table 1: This table shows the mean and standard deviation of the accuracy of the users. Users completed tasks with the most accuracy in the case of augmented animations.

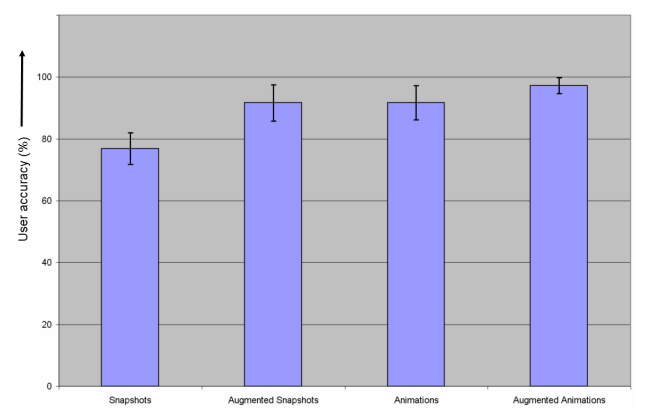

Figure 7: This graph shows accuracy results grouped according to the categories of questions asked. The categories, from left to right, are Snapshots, Augmented Snapshots, Animations, and Augmented Animations. The user accuracy for Augmented Snapshots is better than Snapshots, similarly the accuracy is better for Augmented Animations as compared to Animations.

comparing augmented snapshots (M2) with snapshots (M1). Similarly, the results is significant when comparing augmented animations (M4) with plain animations (M3).

The second metric we used to evaluate the techniques was the time required by the subject to complete each task per visualization technique. Table 4 shows the mean and standard deviation for the four visualization techniques. Figure 8 shows a graphical representation of the timing results. The subjects required more time when viewing snapshots than in the other three cases. Augmented animations helped subjects answer questions faster than just animations. Overall, even though loading an animation took more time, anima-

\begin{tabular}{|c|c|c|c|c|}
\hline $\begin{array}{c}\text { Source } \\
\text { of variation }\end{array}$ & $\begin{array}{c}\text { Sum of } \\
\text { squares }\end{array}$ & DOF & $\begin{array}{c}\text { Mean } \\
\text { squares }\end{array}$ & F \\
\hline between & 6181.0 & 3 & 2060.0 & 12.02 \\
\hline error & 17804.0 & 104 & 171.4 & \\
\hline total & 24004.0 & 107 & & \\
\hline
\end{tabular}

Table 2: This table shows the result of performing the analysis of variance (ANOVA) on the accuracy per user. The probability of these results, assuming the null hypothesis, is less than 0.0001 . 


\begin{tabular}{|c|c|c|c|c|c|}
\hline & & M1 & M2 & M3 & M4 \\
\hline & & 76.851 & 91.67 & 91.67 & 97.22 \\
\hline M1 & 76.851 & - & 14.819 & 14.819 & 20.369 \\
\hline M2 & 91.67 & - & - & 0 & 5.55 \\
\hline M3 & 91.67 & - & - & - & 5.55 \\
\hline M4 & 97.22 & - & - & - & - \\
\hline
\end{tabular}

\begin{tabular}{|c|c|c|c|c|}
\hline $\begin{array}{c}\text { Source } \\
\text { of variation }\end{array}$ & $\begin{array}{c}\text { Sum of } \\
\text { squares }\end{array}$ & DOF & $\begin{array}{c}\text { Mean } \\
\text { squares }\end{array}$ & F \\
\hline between & 16779.0 & 3 & 5593.0 & 16.75 \\
\hline error & 34724.0 & 104 & 333.9 & \\
\hline total & 51503.0 & 107 & & \\
\hline
\end{tabular}

Table 3: The table shows the minimum pairwise difference for accuracy means computed for the Tukey test. Accuracy means are denoted by M1 - snapshots, M2 - augmented snapshots, M3 - animation and M4 - augmented animations.

\begin{tabular}{|c|c|c|c|}
\hline Type of vis. & Mean & $95 \%$ conf.interval & Std. Dev. \\
\hline Snapshots & 54.822 & $47.85-61.80$ & 2.92 \\
\hline $\begin{array}{c}\text { Augmented } \\
\text { Snapshots }\end{array}$ & 22.696 & $15.72-29.67$ & 2.449 \\
\hline Animations & 33.411 & $26.44-40.38$ & 3.107 \\
\hline $\begin{array}{c}\text { Augmented } \\
\text { Animations }\end{array}$ & 26.248 & $19.27-33.22$ & 2.539 \\
\hline
\end{tabular}

Table 4: This table shows the mean, 95\% confidence intervals around the mean and standard deviation for the timing results shown in seconds.

tions seemed to give the user a better understanding of the time-varying nature of the data.

The time required to complete a task in each of the four cases was analyzed using ANOVA. Table 5 shows the result of the ANOVA test on the timing data. The value of $p$ computed using ANOVA was less than 0.0001 which implied that the null hypothesis was rejected. The value of $p$ implies that the result is extremely significant according to the

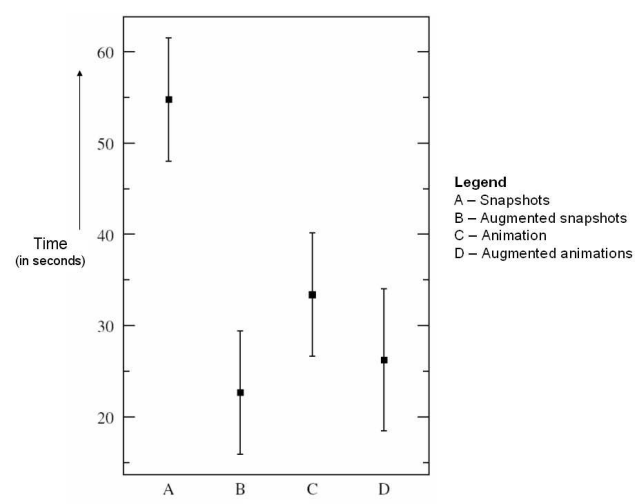

Figure 8: This graph shows the amount of time required by the subjects to complete the task using the four techniques. Users took more time to complete a task using snapshots as compared to all the other techniques. Users also took more time to answer questions using animations as compared to animations augmented with illustration-inspired techniques.
Table 5: This table shows the result of performing the analysis of variance (ANOVA) on the time required by the users to complete a task using all the techniques. The probability of these results, assuming the null hypothesis, is less than 0.0001 .

\begin{tabular}{|c|c|c|c|c|c|}
\hline & & M2 & M4 & M3 & M1 \\
\hline & & 22.696 & 26.248 & 33.411 & 54.822 \\
\hline M2 & 22.696 & - & 3.552 & 10.715 & 32.126 \\
\hline M4 & 26.248 & - & - & 7.163 & 28.574 \\
\hline M3 & 33.411 & - & - & - & 21.411 \\
\hline M1 & 54.822 & - & - & - & - \\
\hline
\end{tabular}

Table 6: The table shows the minimum pairwise difference for timing means computed for the Tukey test. Timing means are denoted by M1 - snapshots, M2 - augmented snapshots, M3 - animation and M4 - augmented animations.

ANOVA test and implies that the use of illustration-inspired techniques clearly helped users complete tasks faster than with just snapshots or animations.

To identify pairwise significance between the timing results, the minimum pairwise difference was calculated using the Tukey test. The computed minimum pairwise difference was $D_{\min }=6.626$ with $Q_{t}=3.9$. For all values of difference of means larger than $D_{\min }$ the pairwise comparisons are considered significant $(p<0.05)$. As can be seen from Table 6 , the results are significant when comparing augmented snapshots (M2) with plain snapshots (M1) as well as when compared with animations (M3). When comparing augmented animations (M4) with animations (M3) the results are significant. A comparison of the augmented animations (M4) and snapshots (M1) too is significant.

The third metric that was used to evaluate the techniques was the user confidence. The subjects were requested to specify a confidence level for each question. Table 7 shows the mean and standard deviation of the confidence obtained for each technique. The confidence was higher for both augmented snapshots and augmented animations, as can be seen in Figure 9. The subjects had the least confidence in their answers for the plain snapshots and low confidence for the plain animations.

Table 8 shows the results of performing the analysis of variance (ANOVA) on user confidence per question. Analyzing the confidence that the subjects had in their results, we found that subjects were more confident in the correctness of their answers when using illustration-inspired techniques. 


\begin{tabular}{|c|c|c|c|}
\hline Type of vis. & Mean & 95\% conf. interval & Std. Dev. \\
\hline Snapshots & 3.6389 & $3.418-3.860$ & 0.088 \\
\hline $\begin{array}{c}\text { Augmented } \\
\text { snapshots }\end{array}$ & 4.0278 & $3.807-4.249$ & 0.078 \\
\hline Animations & 3.7407 & $3.520-3.962$ & 0.103 \\
\hline $\begin{array}{c}\text { Augmented } \\
\text { animations }\end{array}$ & 4.4907 & $4.270-4.712$ & 0.094 \\
\hline
\end{tabular}

Table 7: This table shows the mean, 95\% confidence intervals around the mean as well as standard deviation for the confidence results.

\begin{tabular}{|c|c|c|c|c|}
\hline $\begin{array}{c}\text { Source } \\
\text { of variation }\end{array}$ & $\begin{array}{c}\text { Sum of } \\
\text { squares }\end{array}$ & DOF & $\begin{array}{c}\text { Mean } \\
\text { squares }\end{array}$ & F \\
\hline between & 40.23 & 3 & 13.41 & 5.806 \\
\hline error & 988.5 & 104 & 2.310 & \\
\hline total & 1029.0 & 107 & & \\
\hline
\end{tabular}

Table 8: This table shows the result of performing the analysis of variance (ANOVA) on the confidence per user. The probability of this results, assuming the null hypothesis, is less than 0.0007 .

The value of $p$ from the ANOVA test obtained was $p<$ 0.0007 which according to the ANOVA test is an extremely significant result and rejects the null hypothesis that the users feel equally confident with and without illustration-inspired techniques. The illustration-inspired techniques clearly instill more confidence in the users in both the augmented snapshots and augmented animations.

To identify pairwise significance between the user confidence, the minimum pairwise difference was calculated us-

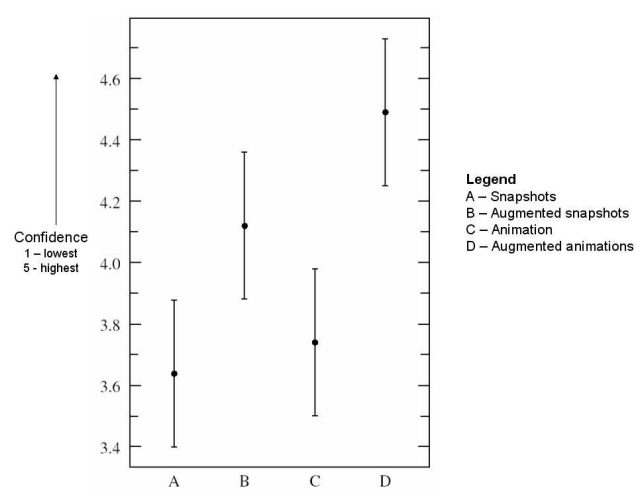

Figure 9: The users were asked to specify their confidence in their answers. This graph shows a representation of the overall confidence that the users had in their answers. As can be seen, users were more confident about their answers for the augmented snapshots and animated animations as compared to plain snapshots or animations.

\begin{tabular}{|c|c|c|c|c|c|}
\hline & & M1 & M3 & M2 & M4 \\
\hline & & 3.6389 & 3.7407 & 4.0278 & 4.4907 \\
\hline M1 & 3.6389 & - & 0.1018 & 0.3889 & 0.8518 \\
\hline M3 & 3.7407 & - & - & 0.2871 & 0.75 \\
\hline M2 & 4.0278 & - & - & - & 0.4629 \\
\hline M4 & 4.4907 & - & - & - & - \\
\hline
\end{tabular}

Table 9: The table shows the minimum pairwise difference for confidence means computed for the Tukey test. Confidence means are denoted by M1 - snapshots, M2 - augmented snapshots, M3 - animation and M4 - augmented animations.

ing the Tukey test. The computed minimum pairwise difference was $D_{\min }=0.688$ with $Q_{t}=3.9$. For all values of difference of means larger than $D_{\min }$ the pairwise comparisons are considered significant $(p<0.05)$. As can be seen from Table 9 , the results are significant when comparing snapshots (M1) and augmented animations (M4). The confidence between augmented animations (M4) and animations (M3) was significant. The pairwise comparison between confidence for augmented snapshots (M2) and snapshots (M1) was not as significant. It means that even though the subjects were more faster and more accurate, they were not as confident using the augmented techniques.

\section{Discussion}

Based on the analysis of the user study, we can say that the illustration-inspired techniques help users track features in three-dimensional time-varying data faster and with more accuracy. The illustrative cues when added to standard visualization techniques such as snapshots or animations provided temporal context.

For synthetic datasets, the users were fast and accurate for all four representations. In the case of real-world data, users were more accurate with the illustration-inspired techniques as can be seen in Figure 10. In particular, for the turbulent vortex data visualized using snapshots, the user accuracy was as low as $30 \%$. Similarly, when visualizing features in the turbulent vortex data using animations, the user accuracy was $66.67 \%$. Overall, the users seem to have struggled with completing the task accurately using standard visualization techniques for real world data. This may be due to the fact that the real world data contains more complex feature movements that the subjects were not able to understand using standard visualization techniques. We observed that the user accuracy using snapshots and animations was lower for complex synthetic datasets containing multiple features and for real-world CFD datasets.

The illustration-inspired techniques when applied to realworld datasets clearly aided the user in accurately identifying the direction of motion of the features. For real-world data, when visualized using augmented snapshots, the user 


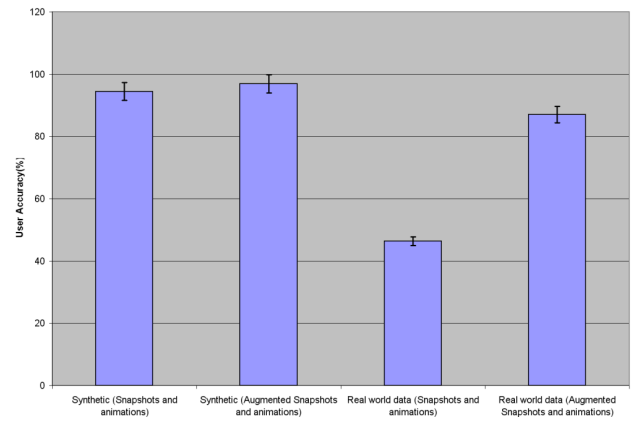

Figure 10: In this graph, the accuracy of the users for synthetic data versus real world data is compared. As can be seen, the accuracy for synthetic data is high for both with and without illustration-inspired techniques. The accuracy for real world data without illustration-inspired techniques is very low whereas the use of illustration-inspired techniques when applied to real world data has boosted the accuracy of the users.

accuracy is much higher at $82 \%$. Subjects performed best (user accuracy $=92.66 \%$ ) with the real world data when visualized using augmented animations. The temporal context and illustrative cues provided using the illustration-inspired techniques seemed to have had a significant impact on the visual tracking abilities of the subjects.

Among the four techniques, the subjects preferred the animations with illustration-inspired techniques the most. This could have been due to the fact that the illustration-inspired techniques augmented animations without distracting the viewer from the main aim of viewing the time-varying phenomenon. The illustrative cues provide important cues to the viewer in a non-invasive manner and clearly help users to complete tasks faster and more accurately than the other techniques. The ability to complete tasks correctly with the use of illustration-inspired techniques seems to have given the users confidence regarding the correctness of their answers.

Our subjective evaluation gave us feedback regarding the users' preferences for the various techniques. Almost all the users (92\%) thought that both the speedlines and the opacity-based techniques helped convey direction better than only snapshots or only animations. Of the two techniques, the users indicated through their answers that they preferred the speedlines technique $(70.8 \%)$ to the opacitybased techniques $(29.2 \%)$. From discussions with domain experts, we think this might be due to misinterpretation of older timesteps actually being data for the current timestep. The amount of opacity variation from one timestep to another needs to be investigated further to avoid such confusion.

\section{Conclusion}

We evaluated previously introduced illustration-inspired techniques by conducting a user study. Analyzing the results of the user study led to the fact that users were able to perform tasks of visually tracking features more accurately and faster using the illustration-inspired techniques than using plain snapshots or animations. Subjects were much more confident of their answers when they used the illustrationinspired techniques. Users performed better at completing tasks with the augmented animations technique as compared to static visualizations. Subjects performed much better on real-world data when using the illustration-inspired techniques as compared to synthetic data, where they were able to perform the task equally well with all the four techniques.

Further evaluation studies in the interactions of multiple such illustration-inspired techniques in a single visualization will provide more guidelines to application domain experts. Occlusions of illustration-inspired techniques by features ahead of them can be further examined to improve the effectiveness of their techniques.

\section{Acknowledgements}

We would like to thank Dr. Deborah Silver and Kristina Bennett for providing the Vortex dataset. We would like to thank all our subjects for participating in the study. We would like to thank the anonymous reviewers for their excellent feedback. This work has been funded by NSF grant numbers 0121288 and 0081581.

\section{References}

[FS98] Fernandez V., Silver D.: Computational fluid dynamics - turbulent vortex dataset: http://www.caip.rutgers.edu/ xswang/feature/index.html, 1998.

[JR05] Joshi A., Rheingans P.: Illustration-inspired techniques for visualizing time-varying data. In Proceedings IEEE Visualization 2005 (2005), pp. 679-686.

[Kit06] KITware I.: Volview: A volume visualization system, 2006.

[Pyl03] Pylyshyn Z. W.: Seeing and Visualizing: Its not what you think (Life and Mind). Bradford Book, 2003.

[SJEG05] Svakhine N., JANG Y., EBert D., GAitheR K.: Illustration and photography inspired visualization of flows and volumes. In Proceedings of IEEE Visualization 2005 (2005), pp. 687-694.

[WS03] Woodring J., Shen H.-W.: Chronovolumes: a direct rendering technique for visualizing time-varying data. In Proceedings of the 2003 Eurographics/IEEE TVCG Workshop on Volume graphics (2003), ACM Press, pp. 27-34. 